\title{
Electrostatic solitons and Alfvén waves generated by streaming instability in electron-positron plasmas
}

\author{
C.-S. Jao ${ }^{1,2, *}$ and L.-N. Hau ${ }^{1,3, \dagger}$ \\ ${ }^{1}$ Institute of Space Science, National Central University, Taoyuan City, Taiwan, Republic of China \\ ${ }^{2}$ DESY, Zeuthen, Germany \\ ${ }^{3}$ Department of Physics, National Central University, Taoyuan City, Taiwan, Republic of China
}

(Received 7 March 2018; published 23 July 2018)

\begin{abstract}
Electron-positron plasmas may be present in laboratory experiments and certain astrophysical environments. Due to the inertia symmetry there have been some debates about whether the nonlinear electrostatic solitons generated easily in electron-proton plasmas may develop in electron-positron plasmas. In this study we show the formation of electrostatic solitons and electromagnetic Alfvén waves in extensive magnetized electron-positron plasma system generated by streaming instabilities based on electromagnetic particle simulations along with fluid theory analyses. In particular, the four-component beam-plasma system may lead to the formation of interlacing electron and positron solitons in the early evolution stage (say, $t<20 \omega_{p}^{-1}$, where $\omega_{p}$ is the plasma frequency) and large-amplitude Alfvén waves in the later phase (say, $t>150 \omega_{p}^{-1}$ ). The magnetic-field perturbations are generated by the beam and firehose-type instabilities associated with temperature anisotropy of $\mathrm{T}_{\|}>\mathrm{T}_{\perp}$ resulting from the parallel plasma heating by the electrostatic instability. It is shown that the growth rates and the dominant wavelengths of both electrostatic and electromagnetic instabilities are consistent with the fluid theory. The coexistence of electrostatic solitons and electromagnetic Alfvén waves with significant magnetic field fluctuations in the same system is a unique feature of electron-positron plasmas and the unified theory behind the formation mechanisms is well addressed in the paper.
\end{abstract}

DOI: 10.1103/PhysRevE.98.013203

\section{INTRODUCTION}

Beam-plasma interaction is a profound process occurring in space, astrophysical, and laboratory plasmas. In our solar system consisting primarily of electrons and protons, electrostatic solitary waves may easily be generated by the streaming instability as shown both observationally and theoretically (see, e.g., Refs. [1-4]). In particular, the inertial asymmetry is essential for the formation of electron solitons associated with hole structures in phase space in electron-proton plasmas. The question is thus raised of whether electrostatic solitons may actually develop in electron-positron plasmas with inertia symmetry (see, e.g., Refs. [5-8]). Based on electrostatic particle simulations along with fluid theory we have recently demonstrated the formation of pair solitons with interlacing positive and negative electric potentials via electron and positron beams streaming in background stationary electron and positron plasmas $[9,10]$. Electrostatic solitons with single polarity in electric potential can also form for certain bump-ontail instabilities with weak beam electrons (positrons) drifting into background electron-positron plasmas [11]. Note that interlacing electron and positron hole structures on the phase space diagram have also been observed in the transition layer of nonrelativistic or weakly relativistic pair shocks for which the electrostatic streaming instability plays the major role in the early stage of the formation processes $[12,13]$.

\footnotetext{
*chun-sung.jao@desy.de

${ }^{\dagger}$ Inhau@jupiter.ss.ncu.edu.tw
}

The importance of studying electron-positron plasmas has two aspects, one being the basic physics associated with simple nonlinear plasma systems and the other being the application of laboratory experiments as well as certain astrophysical environments. In particular, laboratory experiments on beam and plasma interactions in electron-positron plasmas have been performed by using cylindrical and quadrupole Penning traps [14] and high-intensity-laser-induced electron-positron jets [15]. Using the compact magnetic mirror trap, the simultaneous existence of low-energy (a few eV) electrons and positrons in the laboratory has been found to be possible [16]. As for the astrophysical application, theoretical and numerical models have been developed for electron-positron beam-plasma systems present in pulsar magnetospheres to study the magnetic-field fluctuations induced by electromagnetic streaming instabilities associated with the generation mechanism of observed radio emission [17-21]. On the other hand, it has also been proposed that the bunching electric solitons generated by electrostatic streaming instabilities may be radiated away in form of the observed pulsar radio emission [22].

In the past numerical studies, both electrostatic and electromagnetic waves have been observed in unmagnetized or magnetized pair plasma systems subject to counter-streaming instabilities [23-29] or simply generated by thermal fluctuations [30]. Contrary to previous conjectures, we have shown that electrostatic solitons may form in pair plasmas with inertia symmetry [9-10]. In this paper we further extend the previous study to the electromagnetic case with extensive simulation domains based on full particle simulations and fluid theory analyses. It is shown that both electrostatic solitons and large 
amplitude Alfvén waves with distinct wavelengths may exist at different evolutionary stages in the same plasma system subject to the streaming instability. Comparisons between fluid theory and particle simulations are made to provide more insights into the evolution process of streaming instabilities in pair plasmas.

\section{FLUID THEORY}

In this study we consider both the wave propagation and the drifting velocity being along the background magnetic field. Linearization of continuity, momentum, and adiabatic energy equations as well as Maxwell equations yields the following general dispersion relations for streaming instabilities in multicomponent magnetized plasmas:

$$
\begin{gathered}
1=\sum_{\alpha} \frac{\omega_{p, \alpha}^{2}}{\left(\omega-k u_{\alpha 0}\right)^{2}-\gamma_{\alpha} k^{2} v_{\mathrm{th}, \alpha}^{2}}, \\
{\left[\omega^{2}-k^{2} c^{2}-\sum_{\alpha} \frac{\left(\omega-k u_{\alpha 0}\right)^{2}}{\left(\omega-k u_{\alpha 0}\right)^{2}-\omega_{c, \alpha}^{2}} \omega_{p, \alpha}^{2}\right]^{2}} \\
-\left[\sum_{\alpha} \frac{\omega_{c, \alpha}\left(\omega-k u_{\alpha 0}\right)}{\left(\omega-k u_{\alpha 0}\right)^{2}-\omega_{c, \alpha}^{2}} \omega_{p, \alpha}^{2}\right]^{2}=0
\end{gathered}
$$

where $\omega_{c, \alpha} \equiv q_{\alpha} B_{0} / m_{\alpha}$ and $\omega_{p, \alpha} \equiv\left(n_{\alpha 0} q_{\alpha}^{2} / \varepsilon_{o} m_{\alpha}\right)^{1 / 2}$ are the gyro frequency and plasma frequency, respectively. The magnetic field and fluid variables such as the number density and drift velocity with subscript 0 denote the equilibrium quantities. Equation (1) is the dispersion relation for longitudinal or electrostatic modes [9,24], while Eq. (2) is the dispersion relation for transverse or electromagnetic modes which can be separated into $\mathrm{R}$ and $\mathrm{L}$ modes.

We consider the beam-plasma system with a fourcomponent bi-streaming velocity distribution consisting of electrons and positrons streaming in the same direction (denoted by the quantities with subscripts $e 2$ and $p 2$, respectively) relative to the background stationary electrons and positrons (denoted by the quantities with subscripts $e 1$ and $p 1$, respectively). For comparison with our previous results [9,10], all four species are assumed to possess the same number density, thermal velocity $v_{\text {th }}$, and $\gamma$ value (adiabatic exponent) while the two streaming species have the same drift velocity $u_{0}$. The symmetries between electrons and positrons would yield the formation of interlacing electron and positron solitons in electrostatic model $[9,10,12,13]$. The corresponding dispersion relations for the longitudinal mode (electrostatic streaming instability) and the transverse mode (electromagnetic streaming instability) in four-component plasmas are as follows:

$$
\begin{gathered}
\frac{\omega_{p}^{2}}{\omega^{2}-\gamma k^{2} v_{\mathrm{th}}^{2}}+\frac{\omega_{p}^{2}}{\left(\omega-k u_{0}\right)^{2}-\gamma k^{2} v_{\mathrm{th}}^{2}}=1, \\
\frac{\omega^{2}}{\omega_{p}^{2}}-\frac{k^{2} c^{2}}{\omega_{p}^{2}}-\frac{\omega^{2}}{\omega^{2}-\omega_{c}^{2}}-\frac{\left(\omega-k u_{0}\right)^{2}}{\left(\omega-k u_{0}\right)^{2}-\omega_{c}^{2}}=0,
\end{gathered}
$$

where $\omega_{p}^{2}=\omega_{p, e 1}^{2}+\omega_{p, p 1}^{2}=\omega_{p, e 2}^{2}+\omega_{p, p 2}^{2}$. Note that, in the transverse mode presented in Eq. (4), because of the perfect
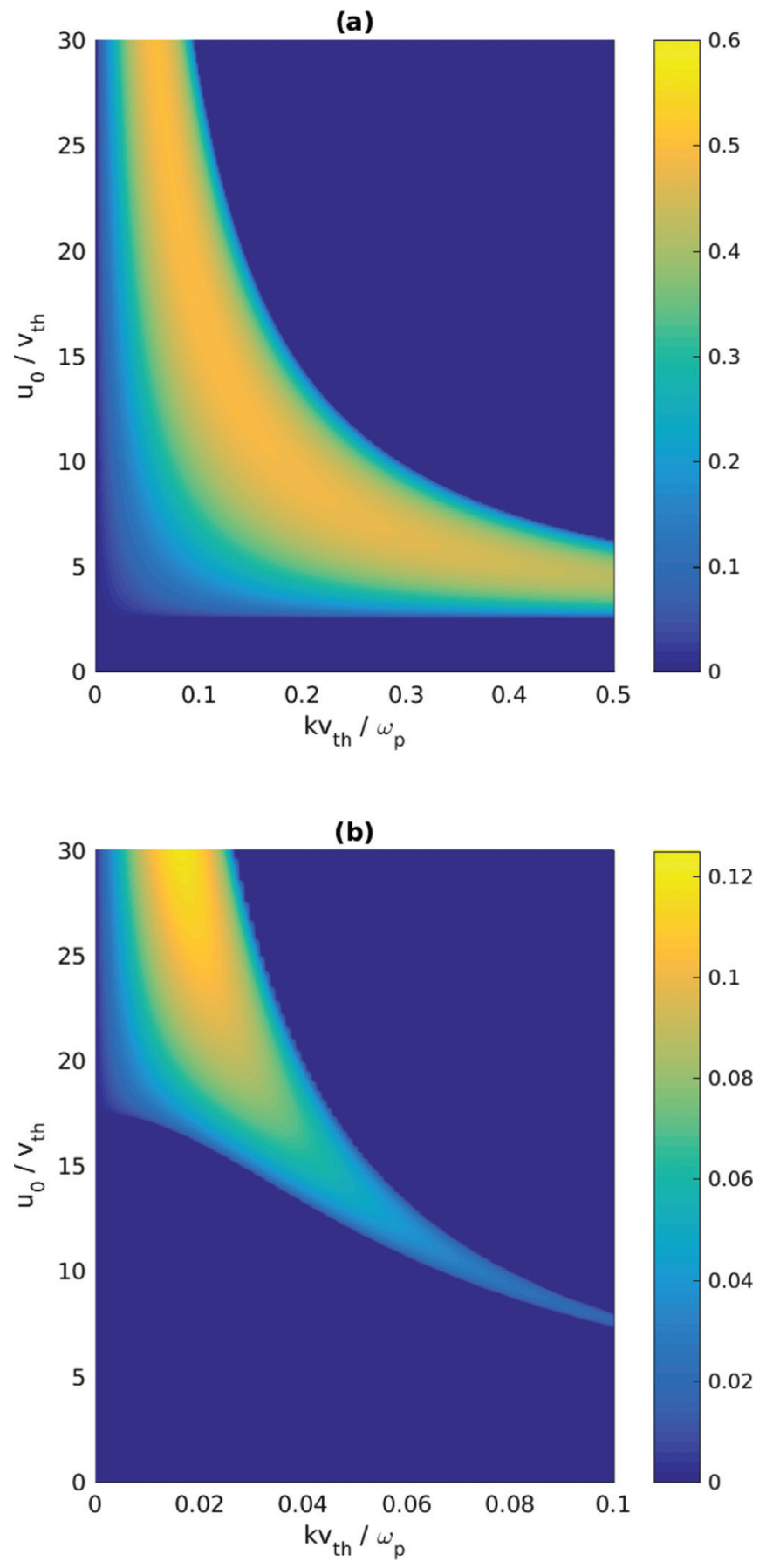

FIG. 1. The imaginary part of the wave frequency $\omega_{i}$ derived from the linear fluid theory as functions of wave number $k$ and $u_{0}$ for (a) the longitudinal mode and (b) the transverse mode.

symmetry between electrons and positrons, the usual $\mathrm{R}$ and $\mathrm{L}$ modes are indistinguishable.

Figure 1 shows the imaginary part of the wave frequency $\omega_{i}$ for longitudinal mode [Fig. 1(a)] and transverse mode [Fig. 1(b)] as functions of wave number $k$ and $u_{0}$ for the parameter value $\omega_{c}=0.4 \omega_{p}$. As expected, for both longitudinal and transverse modes, the growth rates are increased with increasing $u_{0}$ and the wave numbers of the most unstable modes decrease with increasing drift velocity $u_{0}$. Note that, 


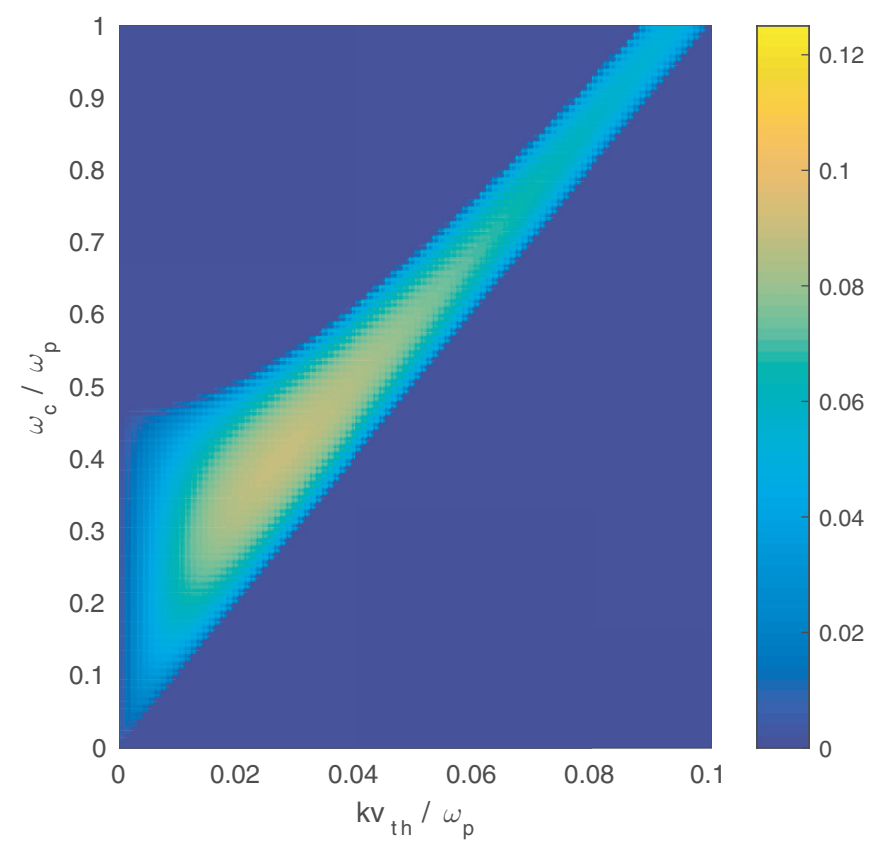

FIG. 2. The imaginary part of the wave frequency $\omega_{i}$ derived from the linear fluid theory as functions of wave number $k$ and $\omega_{c}$ for the transverse mode.

for the same drift velocity $u_{0}$, the growth rates and the corresponding wave numbers of the longitudinal modes are larger than those of the transverse modes. For the comparison with the nonlinear simulation, we particularly note that, for $u_{0}=20 v_{\text {th }}$, the growth rate and wave number for the most unstable longitudinal mode are, respectively, $\omega_{i}=0.497 \omega_{p}$ and $k=0.086 \omega_{p} / v_{\text {th }}$ as compared to $\omega_{i}=0.089 \omega_{p}$ and $k=$ $0.027 \omega_{p} / v_{\text {th }}$ for the transverse mode. This implies that the electromagnetic instability with longer wavelength will occur later than the electrostatic instability. Comparisons with the kinetic simulation results will be made in the following section. Also note that for the most unstable transverse mode we have the relation $\omega_{c}=\omega_{p}-k_{\max } u_{0}$, as a result of the resonance between plasma oscillation and gyro-motion in beam and background plasmas.

Figure 2 shows the imaginary part of the wave frequency $\omega_{i}$ for the transverse mode [Fig. 2(b)] as functions of $k$ and $\omega_{c}$ for the case of $u_{0}=20.0 v_{\text {th }}$. As indicated, the growth rate of the most unstable mode increases with increasing $\omega_{c}$, reaching the maximum value at $\omega_{c} \sim 0.4 \omega_{p}$, and for $\omega_{c} \geqslant 0.4 \omega_{p}$ it decreases with increasing $\omega_{c}$. In particular, for $\omega_{c} \leqslant 0.4 \omega_{p}$, the growth rates are $\omega_{i, \max }=0.034 \omega_{p}, 0.063 \omega_{p}$, $0.083 \omega_{p}$, and $0.089 \omega_{p}$ for $\omega_{c} / \omega_{p}=0.1,0.2,0.3$, and 0.4 , respectively, and the growth rate decreases to $0.085 \omega_{p}$ for $\omega_{c} / \omega_{p}=0.5$ and $0.051 \omega_{p}$ for $\omega_{c} / \omega_{p}=1.0$. Meanwhile, the wave number of the most unstable is shown to increase with increasing $\omega_{c}$; in particular, the corresponding wave numbers are $k=0.012 \omega_{p} / v_{\text {th }}, 0.027 \omega_{p} / v_{\text {th }}$, and $0.094 \omega_{p} / v_{\text {th }}$ for $\omega_{c} / \omega_{p}=$ $0.2,0.4$, and 1.0, respectively. As expected, for $\omega_{c} \approx \omega_{p}$ the wavelengths of the most unstable longitudinal and transverse modes are of the same order.

Note that, because of the one-dimensional assumption with both the wave propagation and drifting velocity being along the background magnetic field, perpendicularly and obliquely (a)

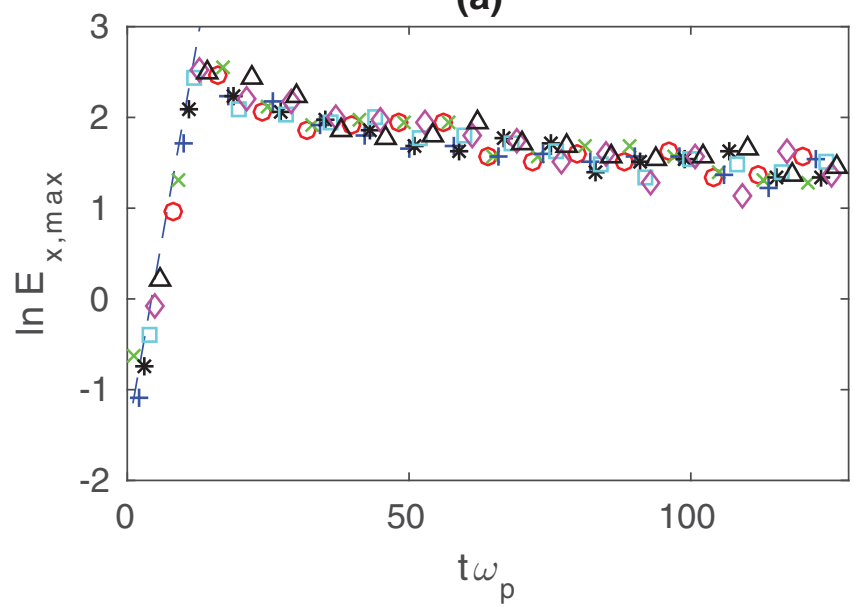

(b)

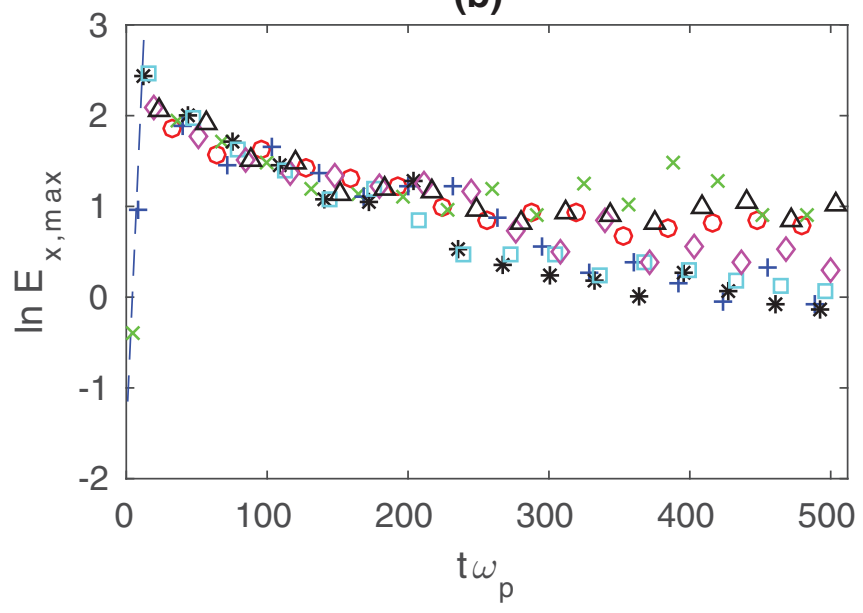

(c)

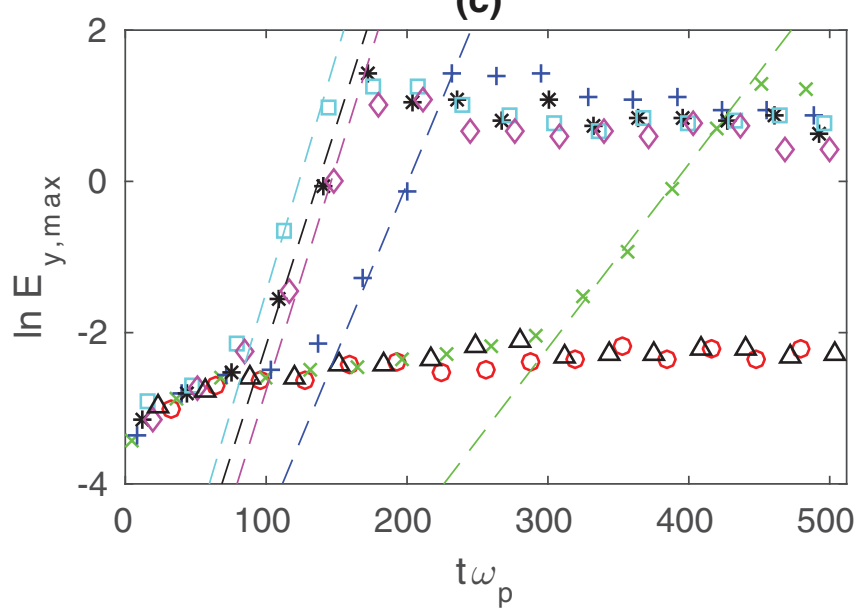

FIG. 3. (a), (b) Time evolution of the logarithm of the longitudinal mode electric field $E_{x}$ and (c) the transverse mode electric field $E_{y}$ with $\omega_{c} / \omega_{p}=0.0$ (circle symbols), 0.1 (cross symbols), 0.2 (plus symbols), 0.3 (star symbols), 0.4 (square symbols), 0.5 (diamond symbols), and 1.0 (triangle symbols) based on the simulation results, respectively. The slope of the corresponding straight lines in each panels are the maximum growth rates of the unstable mode derived from the linear theory.

propagating instabilities such as the filamentation and Weibel instabilities are thus suppressed in our beam-plasma system. It 
(a)

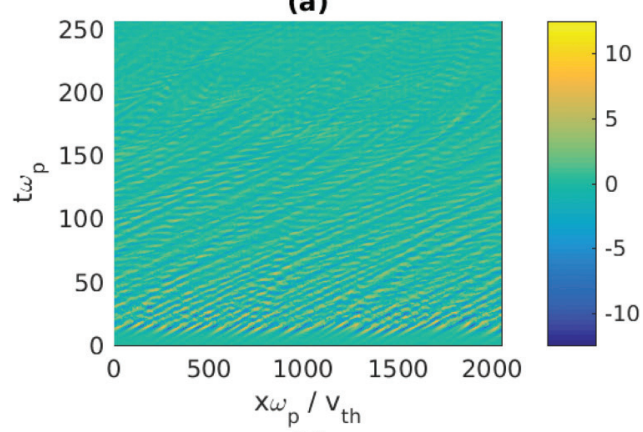

(b)

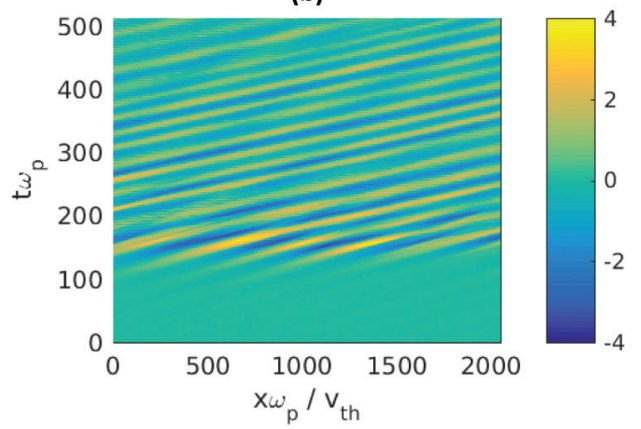

(c)

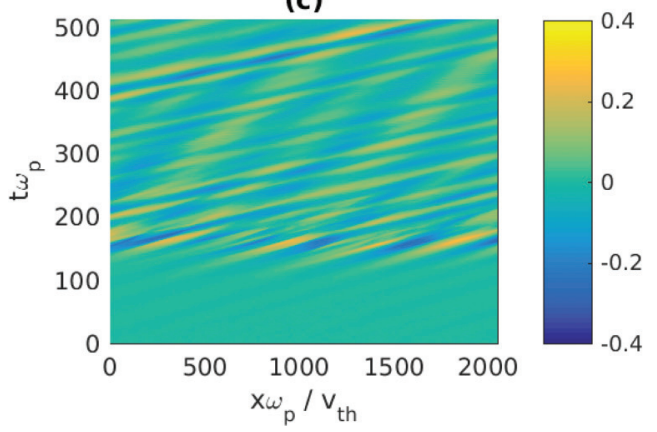

(d)

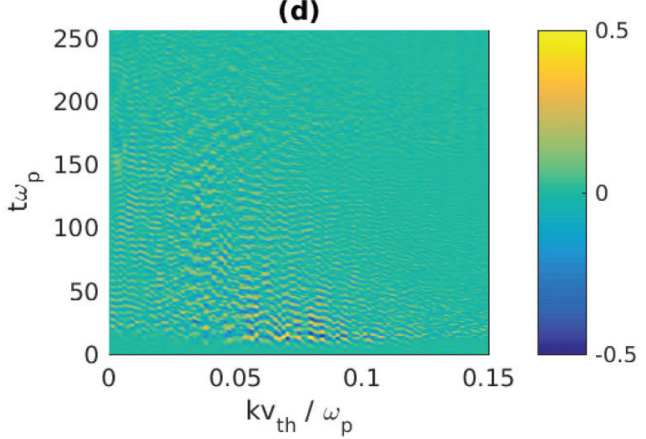

(e)

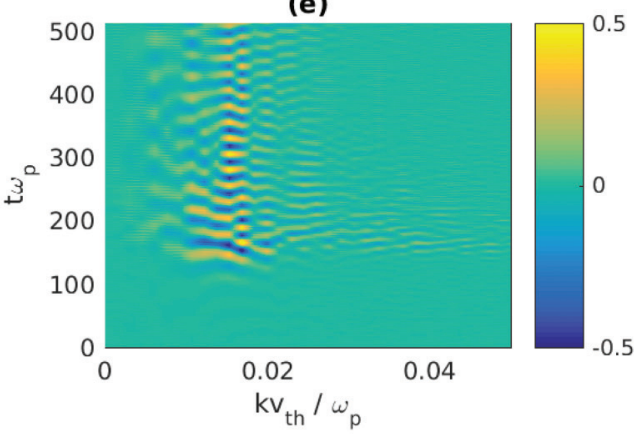

(f)

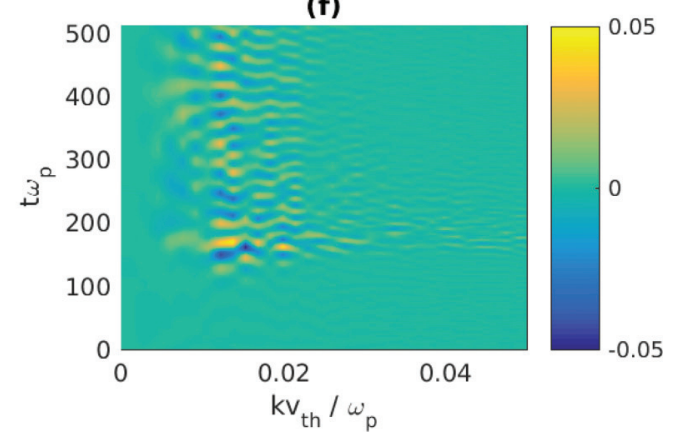

FIG. 4. Temporal and spatial evolution of (a) $E_{x}(x, t)$, (b) $E_{y}(x, t)$, and (c) $B_{y}(x, t)$ and the corresponding time evolution of the wave number for (d) $E_{x}(k, t)$, (e) $E_{y}(k, t)$, and (f) $B_{y}(k, t)$ for the case of $\omega_{c} / \omega_{p}=0.4$.

has been shown that in the non-relativistic regime the growth rate of the filamentation instability may not be comparable to the unstable modes under the present investigation [13,29], while the Weibel instability may compete with the electromagnetic streaming instability for the parameter regime under study $[12,13]$.

\section{KINETIC SIMULATIONS}

To study the nonlinear evolution of electromagnetic streaming instabilities in pair plasmas, a one-dimensional electromagnetic full particle code has been developed. The plasma frequency $\omega_{p}=1.0$ and the thermal velocity of all components $v_{\text {th }}=1.0$ are set as the dimensionless units in the simulation model. The time interval and the grid size are $\Delta t=0.01 \omega_{p}^{-1}$ and $\Delta x=1 \lambda_{D}\left(\lambda_{D}=v_{\mathrm{th}} \omega_{p}^{-1}\right)$, respectively. For all simulation cases shown here the length of the simulation domain is chosen to be $L_{x}=4096 \lambda_{D}$ and the boundary of the simulation system is periodic for both fields and particles. The inertial length of the particles is set to be $\lambda_{i}=32 \lambda_{D}$ in the simulation system. For the initial conditions, the number density distribution is uniform with 512 pairs of electrons and positrons per cell and the initial particle velocity of each species is described by the isotropic Maxwellian distribution. The drifting velocity is $u_{0}=20 v_{\text {th }}$ for both electron and positron beams. In the simulation the background magnetic field and beam drifts are assumed to be in the $x$ direction.

Figure 3 shows the time evolution of the electric field $E_{x}$ [Figs. 3(a) and 3(b)] and $E_{y}$ [Fig. 3(c)] for different ambient magnetic fields $\left(\omega_{c} / \omega_{p}=0.0,0.1,0.2,0.3,0.4,0.5\right.$, and 1.0 as indicated in the caption). The electric components $E_{x}$, $E_{y}$ correspond to longitudinal (electrostatic) and transverse (electromagnetic) modes, respectively. Figure 3(a) shows that in the early stage $\left(t<20 \omega_{p}^{-1}\right)$ longitudinal waves grow with the calculated growth rate $\omega_{i, \max }=0.494 \omega_{p}$. This is in accordance with linear fluid theory [Eq. (3)] and is seen in Fig. 1(a).

After long simulation time $\left(t>150 \omega_{p}^{-1}\right)$ in the magnetized case [Fig. 3(b)] with $\omega_{c} / \omega_{p}=0.1-0.5$, the longitudinal electric field $E_{x}$ is damped, while the transverse electromagnetic field $E_{y}$ [Fig. 3(c)] grows. The slopes of the corresponding straight lines in Fig. 3(c) are the maximum growth rates 
of the unstable electromagnetic mode with the value of $\omega_{i, \max }=0.034 \omega_{p}, 0.063 \omega_{p}, 0.083 \omega_{p}, 0.089 \omega_{p}$, and $0.085 \omega_{p}$ for $\omega_{c} / \omega_{p}=0.1,0.2,0.3,0.4$, and 0.5 , respectively. These agree with linear fluid theory (Fig. 2). The electromagnetic instability appears first with linear growth rate $\omega_{i, \max }=0.089 \omega_{p}$ in the case $\omega_{c} / \omega_{p}=0.4$, as calculated from Eq. (4) and shown in Figs. 1(b) and 2. In the unmagnetized case $\omega_{c} / \omega_{p}=0.0$, it does not occur, as predicted by fluid theory (Fig. 2) and seen in the particle simulations. As for the case of $\omega_{c} / \omega_{p}=1.0$, fluid theory predicts growth of the electromagnetic mode with $\omega_{i, \max }=0.051 \omega_{p}$ (larger than the case of $\omega_{c} / \omega_{p}=0.1$ with $\omega_{i, \max }=0.034 \omega_{p}$ ), which, however, is not seen in the kinetic simulation. We elaborate these results further in the following.

Figure 4 shows the temporal and spatial evolution of longitudinal electric field $E_{x}(x, t)$ [Fig. 4(a)], transverse electric field $E_{y}(x, t)$ [Fig. 4(b)], and magnetic field $B_{y}(x, t)$ [Fig. 4(c)], as well as the corresponding time evolution of wave number for $E_{x}(k, t)$ [Fig. 4(d)], $E_{y}(k, t)$ [Fig. 4(e)], and $B_{y}(k, t)$ [Fig. 4(f)] for the case of $\omega_{c} / \omega_{p}=0.4$. Like in the electrostatic simulations of Jao and Hau [9], solitary electric structures form. In the early stage of the electromagnetic instabilities, they travel in the direction of the electron-positron beams [Fig. 4(a)]. The major longitudinal perturbations have a wave number of $0.05 \sim 0.09 v_{\text {th }} / \omega_{p}$ [Fig. 4(d)], in accord with fluid theory [Fig. 1(a)]. Around $t \sim 150 \omega_{p}^{-1}$ transverse structures form [Fig. 4(b)]. These also travel in the direction of beams. Their major wave numbers are $0.015 \sim 0.022 v_{\text {th }} / \omega_{p}$ [Fig. 4(e)] corresponding to wavelengths of $285 \sim 420 \lambda_{D}$, much larger than those of the longitudinal perturbations (which have wavelengths of $50 \sim 80 \lambda_{D}$ ), which is consistent with fluid theory. For smaller simulation domains, say, $L_{x}=256 \lambda_{D}$, the electromagnetic streaming instability, however, would not develop and the electrostatic solitons formed by the electrostatic instability would remain stable in the simulation system. The electromagnetic instability is associated with the magneticfield perturbation in the simulation system [Fig. 4(c)] with the same wave number of $0.015 \sim 0.022 v_{\text {th }} / \omega_{p}$ [Fig. 4(f)]. The magnetic-field hodograms at $t=168 \omega_{p}^{-1}$ [Fig. 4(a)] and $t=256 \omega_{p}^{-1}\left[\right.$ Fig. 4(b)] for the case of $\omega_{c} / \omega_{p}=0.4$ are shown in Fig. 5. Starting with the value of $\left(B_{y}, B_{z}\right)$ from $x=0$ (circle symbol), there is no purely linear or circular polarization at both $t=168 \omega_{p}^{-1}$ [Fig. 5(a)] and $t=256 \omega_{p}^{-1}$ [Fig. 5(b)]; instead, linear, right-hand circular, and left-hand circular polarizations are observed in different regions of the simulation domain.

Figure 6 shows the phase space of the particles at $t=64 \omega_{p}^{-1}$ and $t=168 \omega_{p}^{-1}$ for the cases of $\omega_{c} / \omega_{p}=0.0$ [Figs. 6(a) and $6(\mathrm{~d})$ ], $\omega_{c} / \omega_{p}=0.4$ [Figs. 6(b) and 6(e)], and $\omega_{c} / \omega_{p}=1.0$ [Figs. 6(c) and 6(f)]. As indicated, at $t=64 \omega_{p}^{-1}$ the cases shown in Figs. 6(a)-6(c) all exhibit the interlacing electron and positron holes in phase space, which are identical to the oneand two- dimensional electrostatic simulation results $[9,10]$. Similar phase space structures have also been seen in the formation processes of nonrelativistic or weakly relativistic pair shocks $[12,13]$. As for the case of $\omega_{c} / \omega_{p}=0.4$ shown in Fig. 6(e), at $t=168 \omega_{p}^{-1}$ the hole structures that form are obviously destroyed on the phase space diagram after the electromagnetic instability takes place (square symbols in [Fig. 3(c)]. Since the electromagnetic instability does not occur for the cases of $\omega_{c} / \omega_{p}=0.0$ [circles in Fig. 3(c)] and (a)

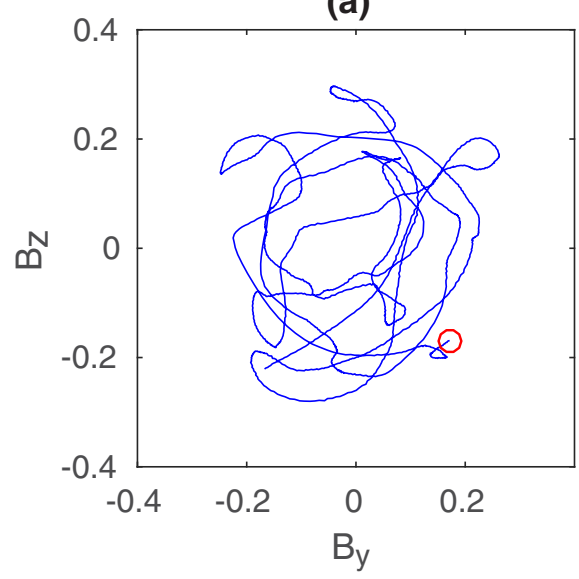

(b)

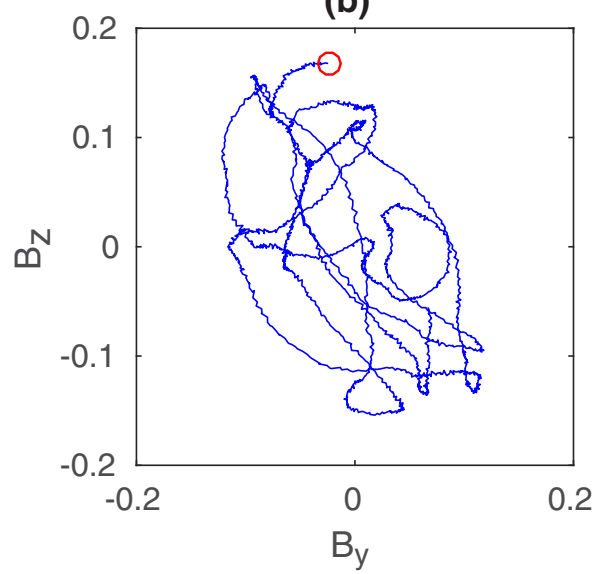

FIG. 5. The magnetic field hodograms at (a) $t=168 \omega_{p}^{-1}$ and (b) $t=256 \omega_{p}^{-1}$ for the case of $\omega_{c} / \omega_{p}=0.4$. The circle symbol in each panels denotes the value of $\left(B_{y}, B_{z}\right)$ at $x=0$.

$\omega_{c} / \omega_{p}=1.0$ [triangles in Fig. 3(c)], the formed interlacing electron and positron hole structures may still exist in the simulation system at $t=168 \omega_{p}^{-1}\left[\right.$ Fig. 6(d)] for $\omega_{c} / \omega_{p}=0.0$ and [Fig. 6(f)] for $\omega_{c} / \omega_{p}=1.0$. The time evolution of the electric-potential drop $\Delta \phi$ in the simulation system is shown in Fig. 7 for various cases with the same drift velocity. In the early stage (say, $t<100 \omega_{p}^{-1}$ ) all three cases are nearly the same while $\Delta \phi$ is decreased significantly for the case $\omega_{c} / \omega_{p}=0.4$ (cyan curve) after $t \sim 150 \omega_{p}^{-1}$, which is associated with the development of electromagnetic instability and the destruction of electron-positron hole structures. Note that the $\Delta \phi$ curves for $\omega_{c} / \omega_{p}=0.0$ (red curve) and $\omega_{c} / \omega_{p}=1.0$ (black curve) cases separate in the period of $t=200 \sim 300 \omega_{p}^{-1}$, coincident with the time evolution of the transverse electric field $E_{y}$ shown in Fig. 3(c), and the stable existence of $\Delta \phi$ in both cases corresponds to the electron-positron holes shown in Figs. 6(d) and $6(f)$.

Figure 8 shows the particle velocity distributions at various times for the cases of $\omega_{c} / \omega_{p}=0.0$ [Fig. 8(a)], $\omega_{c} / \omega_{p}=0.4$ [Fig. 8(b)], and $\omega_{c} / \omega_{p}=1.0$ [Fig. 8(c)]. Note that we only present the velocity distribution of positrons in Fig. 8 since the distributions are almost identical for both electrons and positrons. As indicated, the velocity distributions after the electrostatic instability has taken place at, say, $t=64 \omega_{p}^{-1}$ (dashed curve in all panels), show no apparent differences 

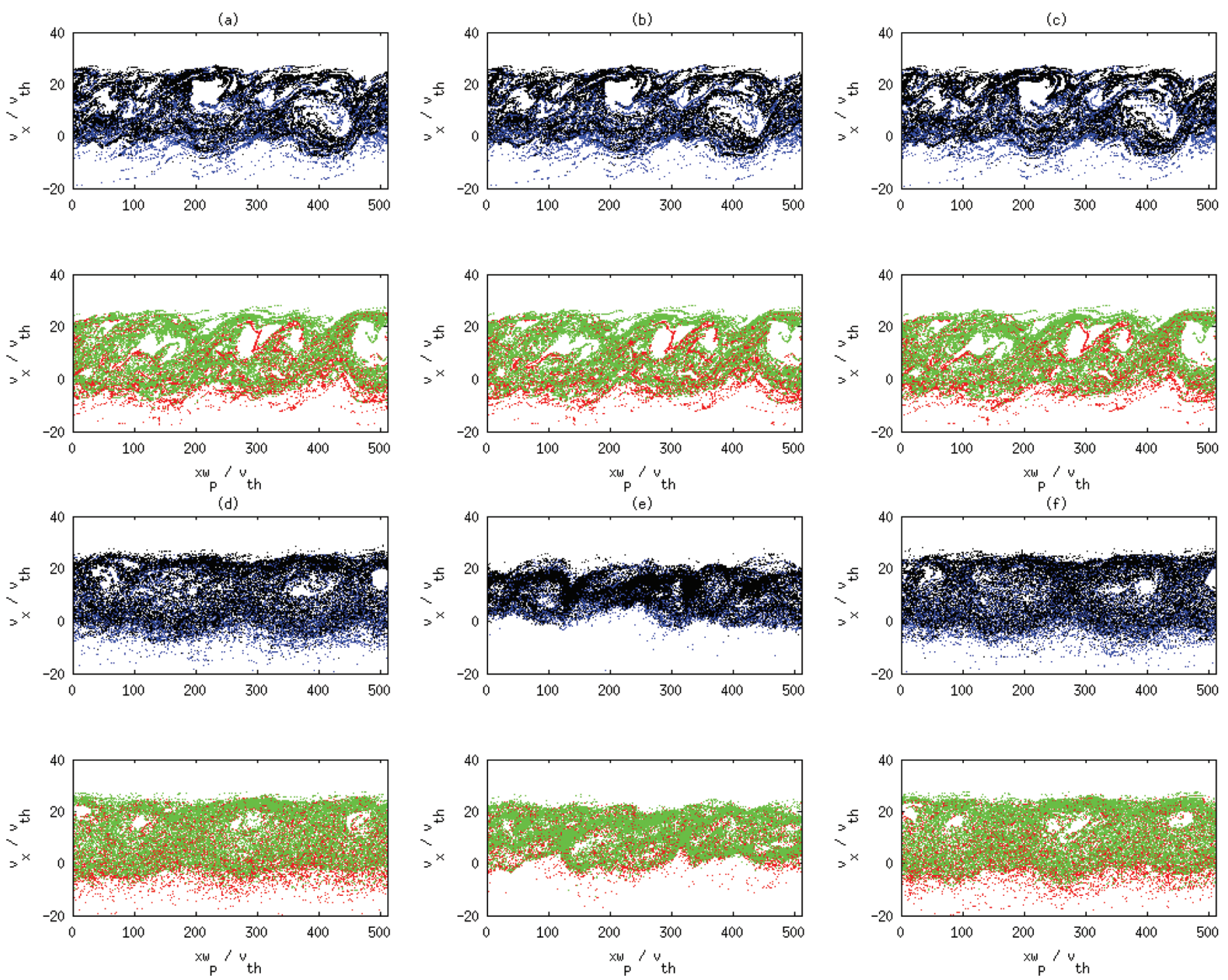

FIG. 6. Phase space of the particles for the cases of $\omega_{c} / \omega_{p}=0.0$ at (a) $t=64 \omega_{p}^{-1}$ and (d) $t=168 \omega_{p}^{-1}, \omega_{c} / \omega_{p}=0.4$ at (b) $t=64 \omega_{p}^{-1}$ and (e) $t=168 \omega_{p}^{-1}$, and $\omega_{c} / \omega_{p}=1.0$ at (c) $t=64 \omega_{p}^{-1}$ and (f) $t=168 \omega_{p}^{-1}$. The blue, black, red, and green dots denote background positrons, beam positrons, background electrons, and beam electrons, respectively.

among the three cases. It is seen that the velocity distributions for parallel (top panel) and perpendicular (middle and bottom

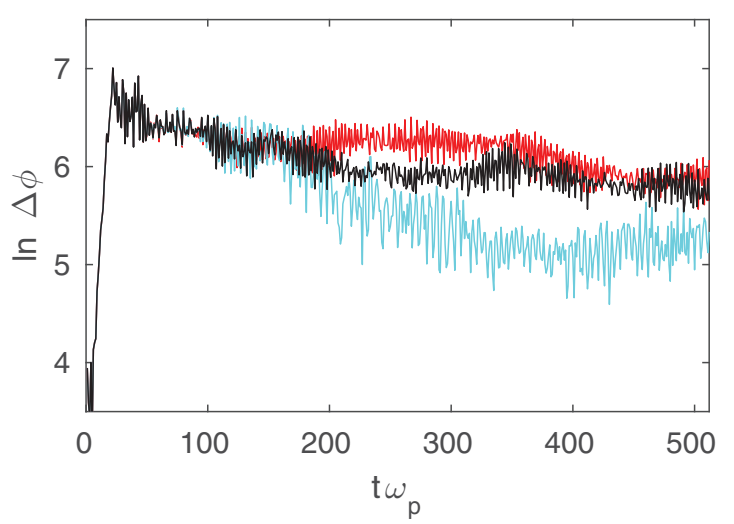

FIG. 7. Time evolution of the logarithm of the electric potential drop $\Delta \phi$ for the simulation cases with $\omega_{c} / \omega_{p}=0.0$ (red curve), 0.4 (cyan curve), and 1.0 (black curve). panels) components with respect to the background magnetic field and drifting direction are distinct in that temperature or pressure anisotropy with $p_{\|}>p_{\perp}$ develops at $t=64 \omega_{p}^{-1}$ (dashed curve) in all three cases after the electrostatic instability has occurred. The built-up pressure anisotropy is reduced at $t=256 \omega_{p}^{-1}$ by the electromagnetic instability as evidenced by the plasma heating in the perpendicular direction (dot-dashed curve in panel $\mathrm{b}$ for the case of $\omega_{c} / \omega_{p}=0.4$ ). On the other hand, for the cases of $\omega_{c} / \omega_{p}=0.0$ [Fig. 8(a)] and $\omega_{c} / \omega_{p}=1.0$ [Fig. 8(c)] for which the electromagnetic instability does not take place, the velocity distributions and the temperature anisotropy remain unchanged from $t=64 \omega_{p}^{-1}$ (dashed curve) to $t=256 \omega_{p}^{-1}$ (dot-dashed curve).

As shown already, according to the linear fluid theory both cases of $\omega_{c} / \omega_{p}=0.1$ and $\omega_{c} / \omega_{p}=1.0$ are unstable to the electromagnetic instability with slightly larger growth rate for the case of $\omega_{c} / \omega_{p}=1.0$. However, in the nonlinear simulations the electromagnetic instability develops for the case of $\omega_{c} / \omega_{p}=0.1$ but not for the case of $\omega_{c} / \omega_{p}=1.0$. The question is thus raised of what are the conditions for the 
(a)
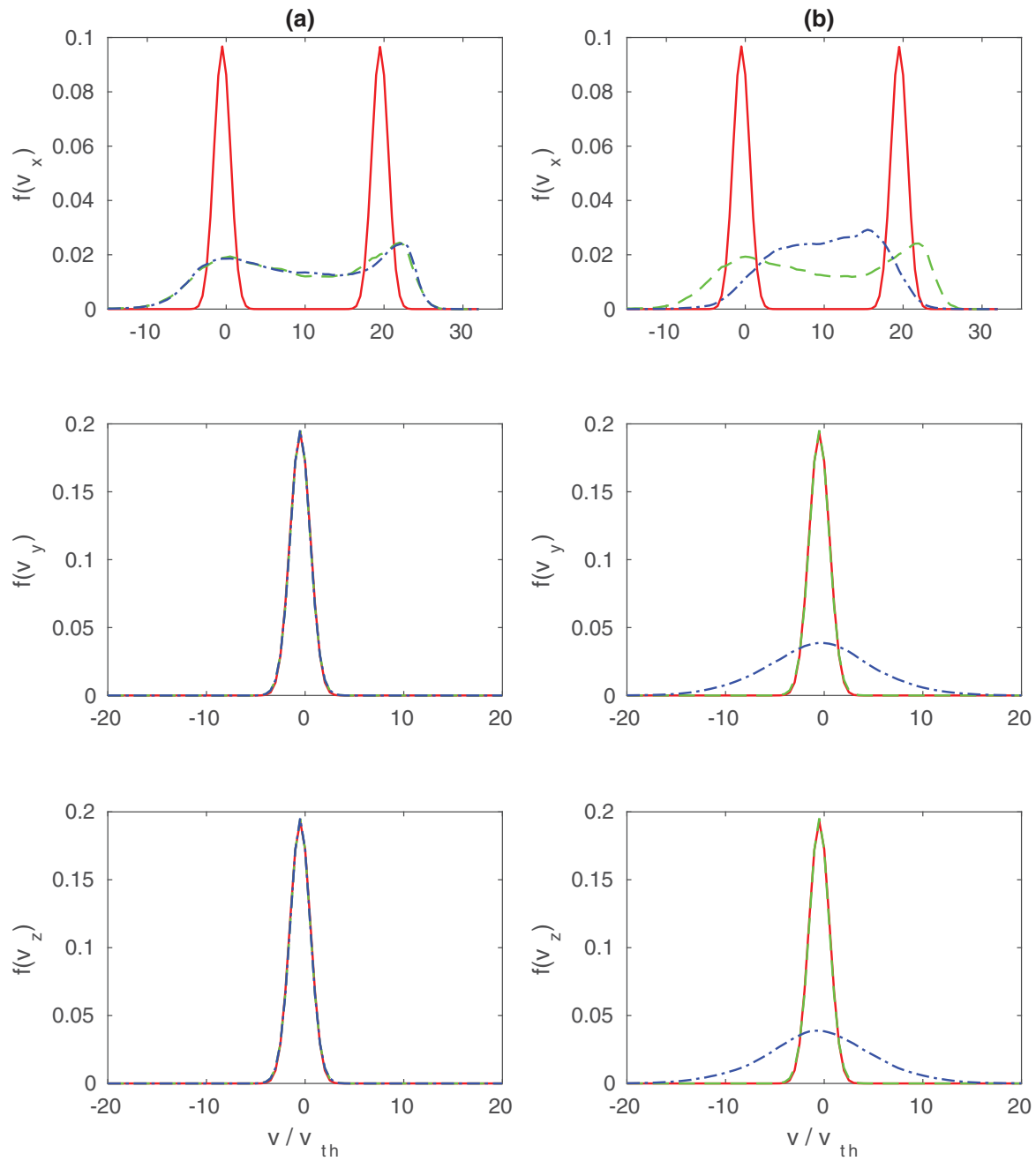

(c)
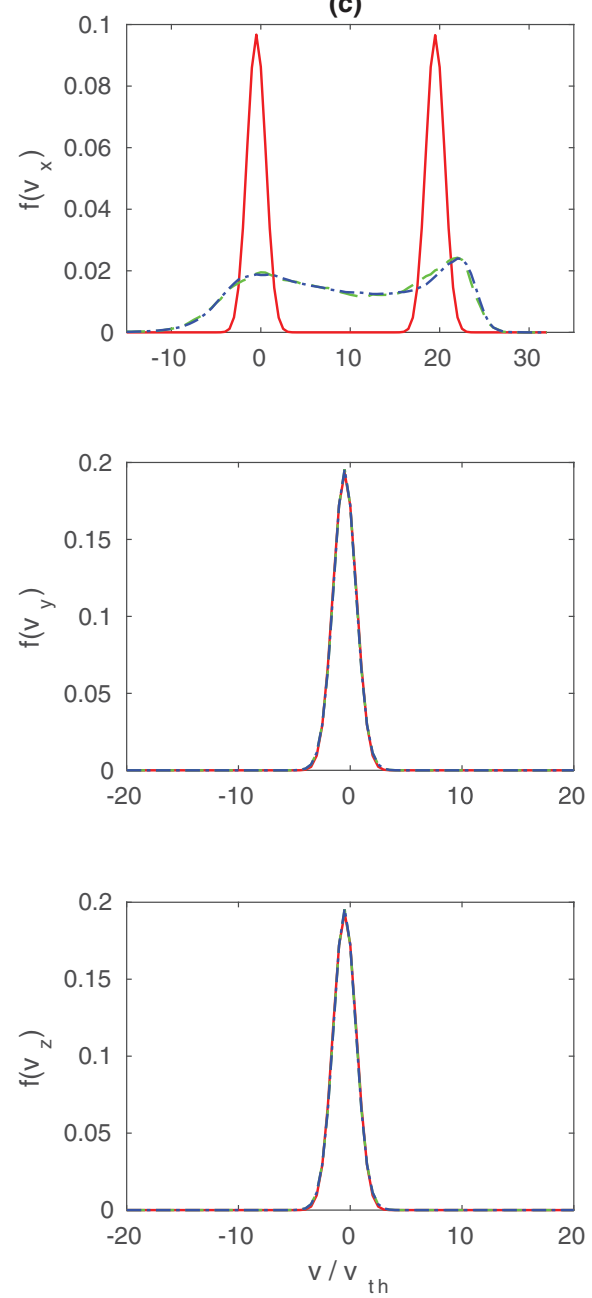

FIG. 8. Velocity distributions of positrons at $t=0$ (solid curve), $t=64 \omega_{p}^{-1}$ (dashed curve), and $t=256 \omega_{p}^{-1}$ (dot-dashed curve) for the cases of (a) $\omega_{c} / \omega_{p}=0.0$, (b) $\omega_{c} / \omega_{p}=0.4$, and (c) $\omega_{c} / \omega_{p}=1.0$. The panels from top to bottom denote the velocity distributions $f\left(v_{x}\right), f\left(v_{y}\right)$, and $f\left(v_{z}\right)$, respectively.

development of electromagnetic instability in the pair plasma system under study. The above analyses have shown that the temperature anisotropy associated with the parallel plasma heating is likely to be the free energy for the electromagnetic instability. In particular, following the magnetohydrodynamic (MHD) theory and earlier kinetic simulations for proton firehose instability the dimensionless quantity of $S=\beta_{\|}-\beta_{\perp}$, where $\beta_{\|}=P_{\|} /\left(B^{2} / 2 \mu_{0}\right)$ and $\beta_{\perp}=P_{\perp} /\left(B^{2} / 2 \mu_{0}\right)$, is used to measure the degree of pressure anisotropy in the simulations [31,32]. Figure 9 shows the time evolution of plasma betas (top and middle panels are for background and beam positrons, respectively) and magnetic field perturbation $\delta B^{2} / B_{0}^{2}$ (bottom panels) for the cases of $\omega_{c} / \omega_{p}=0.2$ [Fig. 9(a)], $\omega_{c} / \omega_{p}=$ 0.4 [Fig. 9(b)] and $\omega_{c} / \omega_{p}=1.0$ [Fig. 9(c)]. It is seen that, for $t<20 \omega_{p}^{-1}$, the plasma beta $\beta_{\|}$(solid curve) and the pressure anisotropy $S$ are increased in all cases, caused by the electrostatic instability. In particular, the cases of $\omega_{c} / \omega_{p}=0.2$ [Fig. 9(a)] and $\omega_{c} / \omega_{p}=0.4$ [Fig. 9(b)] have much larger $\beta_{\|}$and $S=\beta_{\|}-\beta_{\perp}$ values as compared to the case of $\omega_{c} / \omega_{p}=1.0$ [Fig. 9(c)] and the decreasing of $\beta_{\|}, S$ and the increasing of $\beta_{\perp}$ are associated with the occurrence of electromagnetic instability corresponding to the variation of the velocity distribution shown in Fig. 8(b). It is apparent that, for the case of $\omega_{c} / \omega_{p}=1.0$, the $S=\beta_{\|}-\beta_{\perp}$ value is too small to trigger the firehose-type instability. The saturated $S$ value (dot-dashed curve) for both cases of $\omega_{c} / \omega_{p}=0.2$ [Fig. 9(a)] and $\omega_{c} / \omega_{p}=0.4$ [Fig. 9(b)] is about 0.2, which is much smaller than the criterion of $\beta_{\|}-\beta_{\perp}=2$ for the fluid firehose instability, an indication of significant resonance effects [31]. Note that all cases shown in Fig. 9 have the same growth rate for electrostatic instability, yielding similar thermal pressure $p_{\|}$and $p_{\perp}$. As a result, $\beta_{\|}-\beta_{\perp}$ is smaller for larger $\omega_{c} / \omega_{p}$, which explains the small value of $S$ in the case of $\omega_{c} / \omega_{p}=1.0$. The magnetic-field perturbations shown in the third row of Fig. 9 exhibit coincident features with the time evolution of plasma betas and the degree of pressure anisotropy. In particular, for the unstable cases [Figs. 9(a) and 9 (b)], $\delta B^{2} / B_{0}^{2}$ is abruptly increased prior to $S=\beta_{\|}-\beta_{\perp}$ reaching to certain stable critical values at $t \approx 200 \omega_{p}^{-1}$. The abrupt increase of $\delta B^{2} / B_{0}^{2}$ followed by a sudden drop to a low saturated value is similar to the particle simulations of firehose-type instabilities in electron-proton plasmas [31]. 
(a)
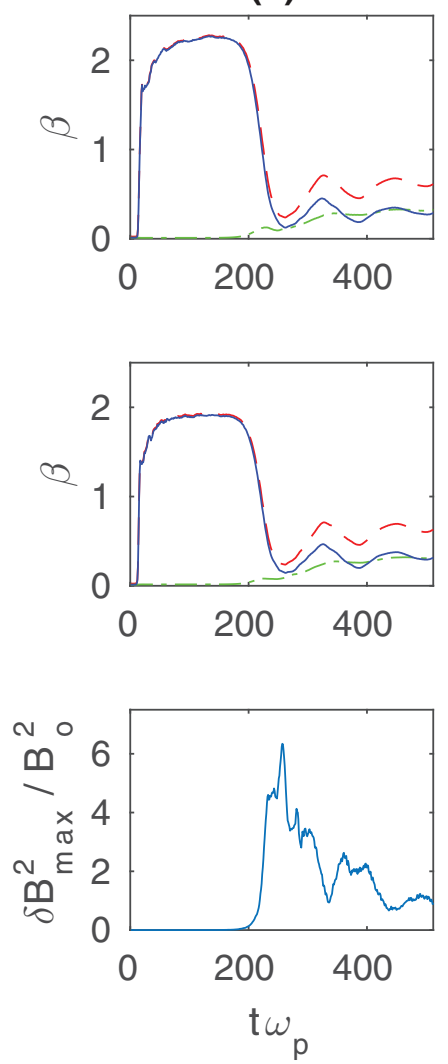

(b)
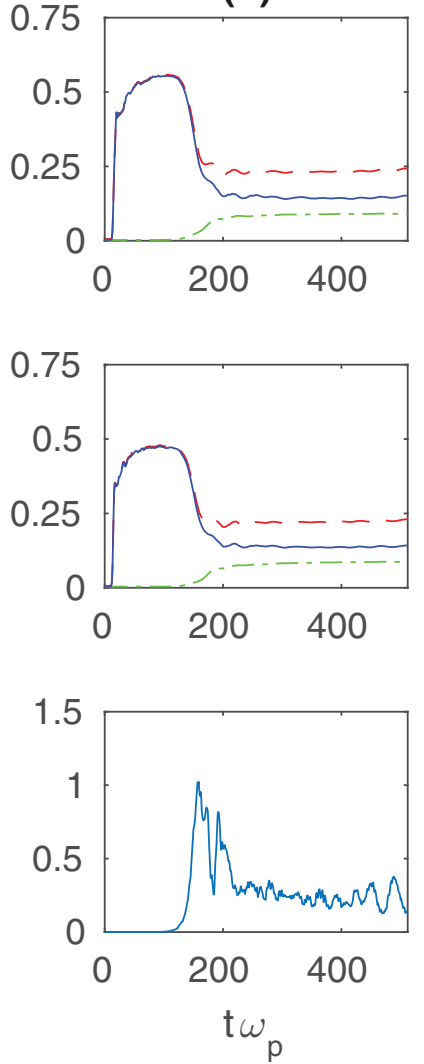

(c)
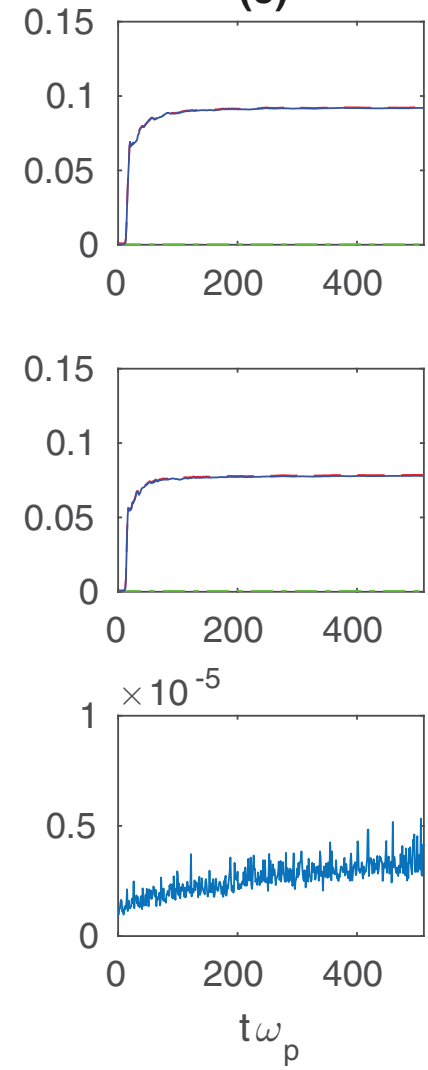

FIG. 9. Time evolution of plasma beta $\beta$ for background positrons (top panels) and beam positrons (middle panels) and the quantity $\delta B^{2} / B_{0}^{2}$ (bottom panels) for the cases of (a) $\omega_{c} / \omega_{p}=0.2$, (b) $\omega_{c} / \omega_{p}=0.4$, and (c) $\omega_{c} / \omega_{p}=1.0$. The solid, dashed, and dot-dashed curves in top and middle panels denote $\left(\beta_{\|}-\beta_{\perp}\right), \beta_{\|}$, and $\beta_{\perp}$, respectively.

These analyses show that the electromagnetic instability is attributed to the degree of pressure anisotropy, resulting from electrostatic streaming instability and measured by $S=$ $\beta_{\|}-\beta_{\perp}$. The properties of the magnetic field perturbations associated with the electromagnetic instability shown above have several similarities to the Alfvén waves in the context of MHD theory. In particular, the Alfvén waves are subject to firehose instability with frequency being much smaller than the gyro frequency. Additionally, the electric field is nearly perpendicular to the magnetic field after the development of electromagnetic instability obeying more or less the frozen-influx condition which is an important mechanism for generating large-amplitude magnetic-field fluctuations.

As shown in our previous study, it is also possible to form electrostatic solitary structures with single polarity in an electric potential via weak-beam electrons (positrons) drifting into background electron-positron plasmas, the so-called bump-on-tail instabilities [11]. We have attempted to carry out similar experiments as shown above for the bump-ontail instability in electron-positron plasmas and found that electromagnetic instability is unlikely to be induced by the weak beam-plasma interaction. Specifically, the heating in beam components associated with the electrostatic instability is not sufficient to trigger firehose-type instabilities in the system.

\section{CONCLUSION}

In this paper we have reported the formation of electrostatic pair solitons generated by streaming instabilities in electron-positron magnetized plasmas based on electromagnetic particle simulations with extensive modeling spatial domain. Due to the significant parallel heating temperature or pressure anisotropy may develop which in turn triggers the electromagnetic firehose-type instabilities associated with significant growth of magnetic-field perturbations. We have analyzed both instabilities based on linear plasma fluid theory and compared with the kinetic simulation results in terms of the growth rate and the wavelength of the unstable modes, which show remarkable agreement. The condition for electromagnetic instability derived from the linear fluid theory, however, is only a necessary but not a sufficient condition. It is shown that in the kinetic simulations the degree of pressure anisotropy in terms of $S=\beta_{\|}-\beta_{\perp}$ value, which is less for stronger background magnetic field, is required to develop the electromagnetic firehose-type instability. In the final stage of nonlinear evolution the magnetic field is dissipated with significant reduction of temperature anisotropy as a result of wave particle interactions. For certain parameter regimes both electrostatic electron and positron solitons as well as electromagnetic Alfvén waves thus may occur at different 
times under the same plasma system. This feature is unlikely to occur in electron-proton system with very distinct inertial masses and is a significant result for electron-positron plasmas with inertia symmetry.

\section{ACKNOWLEDGMENT}

This research is supported by the Ministry of Science and Technology of the Republic of China (Taiwan) under Grant No. MOST-106-2111-M-008-026 to National Central University.
[1] H. Matsumoto et al., Geophys. Res. Lett. 21, 2915 (1994).

[2] R. E. Ergun, C. W. Carlson, J. P. McFadden, F. S. Mozer, L. Muschietti, I. Roth, and R. J. Strangeway, Phys. Rev. Lett. 81, 826 (1998).

[3] H. Schamel, Phys. Plasmas 19, 020501 (2012).

[4] I. H. Hutchinson, Phys. Plasmas 24, 055601 (2017).

[5] G. P. Zank and R. G. Greaves, Phys. Rev. E 51, 6079 (1995).

[6] B. Eliasson and P. K. Shukla, Phys. Rev. E 71, 046402 (2005).

[7] F. Verheest, Phys. Plasmas 13, 082301 (2006).

[8] M.-H. Woo et al., Phys. Plasmas 24, 042903 (2017).

[9] C.-S. Jao and L.-N. Hau, Phys. Rev. E 86, 056401 (2012).

[10] C.-S. Jao and L.-N. Hau, New J. Phys. 17, 053047 (2015).

[11] C.-S. Jao and L.-N. Hau, Phys. Rev. E 89, 053104 (2014).

[12] M. E. Dieckmann and A. Bret, J. Plasma Phys. 83, 905830104 (2017).

[13] M. E. Dieckmann and A. Bret, Mon. Not. R. Astron. Soc. 473, 198 (2018).

[14] C. P. Ridgers, C. S. Brady, R. Duclous, J. G. Kirk, K. Bennett, T. D. Arber, A. P. L. Robinson, and A. R. Bell, Phys. Rev. Lett. 108, 165006 (2012).

[15] H. Higaki et al., New J. Phys. 19, 023016 (2017).

[16] J. Warwick, T. Dzelzainis, M. E. Dieckmann, W. Schumaker, D. Doria, L. Romagnani, K. Poder, J. M. Cole, A. Alejo, M. Yeung,
K. Krushelnick, S. P. D. Mangles, Z. Najmudin, B. Reville, G. M. Samarin, D. D. Symes, A. G. R. Thomas, M. Borghesi, and G. Sarri, Phys. Rev. Lett. 119, 185002 (2017).

[17] V. V. Usov, Astrophys. J. 320, 333 (1987).

[18] V. N. Ursov and V. V. Usov, Astrophys. Space Sci. 140, 325 (1988).

[19] Y. E. Lyubarskii, Astron. Astrophys. 261, 544 (1992).

[20] Y. E. Lyubarskii, Astron. Astrophys. 265, L33 (1992).

[21] E. Asseo and G. I. Melikidze, Mon. Not. R. Astron. Soc. 301, 59 (1998).

[22] G. I. Melikidze, J. A. Gil, and A. D. Pataraya, Astrophys. J. 544, 1081 (2000).

[23] C. S. Choi et al., Astrophys. Space Sci. 145, 241 (1998).

[24] J. Zhao et al., Phys. Plasmas 1, 103 (1994).

[25] Y. Kazimura et al., Astrophys. J. 498, L183 (1998).

[26] T. Haruki, and J. I. Sakai, Phys. Plasmas 10, 392 (2003).

[27] S. Saito and J. I. Sakai, Astrophys. J. 602, L41 (2004).

[28] S. Saito and J. I. Sakai, Phys. Plasmas 11, 859 (2004).

[29] L. O. Silva et al., Astrophys. J. 596, L121 (2003).

[30] R. A. López et al., Astrophys. J. 810, 103 (2015).

[31] S. P. Gary et al., J. Geophys. Res. 103, 14567 (1998).

[32] B.-J. Wang and L.-N. Hau, J. Geophys. Res. 108, 1463 (2003). 